\title{
Influenza as a cause of severe acute respiratory infection through sentinel surveillance at national and provincial hospitals in Vietnam
}

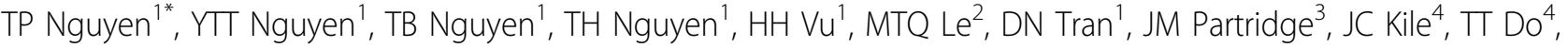 \\ HT Nguyen ${ }^{5}$
}

From 3rd International Conference on Prevention and Infection Control (ICPIC 2015)

Geneva, Switzerland. 16-19 June 2015

\section{Introduction}

Since 2011 a National Influenza Surveillance System in Vietnam has conducted SARI surveillance to collect information and inform control and prevention strategies.

\section{Objectives}

To determine the burden of influenza related SARI by gender and age.

\section{Methods}

Selected patients met the WHO's SARI definition (an acute respiratory infection with history of fever or measured temperature $\geq 38 \mathrm{C}^{\circ}$; cough onset within the last 10 days; and requires hospitalization), were enrolled at four hospitals. Demographic, clinical data and one respiratory swab specimen were collected. Specimens were tested by PCR for influenza virus.

\section{Results}

From March 2011 to April 2013, a total of 2,876 SARI patients were enrolled. The median age was 16 years (IQR 1-34 years). Of all enrolled patients, 1392 (48.4\%) were children $<15$ years old and $1482(51.5 \%)$ were male. 297 (10.3\%) of 2,876 SARI cases were positive for influenza virus by RT-PCR. Positive rates by age group in years were 0 to $<5(26.9 \%), 5$ to $<15(1.7 \%), 15$ to $<25$ (19.5\%), 25 to $<35$ (25.3\%), 35 to $<45$ (7.7\%), 45 to $<55$ (6.7\%), 55 to $<65$ (3.4\%) and $\geq 65(8.8 \%)$. Of the 297 influenza positive samples, 145(48.8\%) were B, 85 (28.6\%) were $\mathrm{A} / \mathrm{H} 3 \mathrm{~N} 2$, and 67 (22.6\%) were $\mathrm{A} / \mathrm{H} 1 \mathrm{~N} 1 \mathrm{pdm} 09$. Among the influenza positive patients, 45 (15.1\%) were

'Epidemiology, National Institute of Hygiene and Epidemiology, Hanoi, Vietnam

Full list of author information is available at the end of the article hospitalized in the ICU. At least one underlying health condition was reported by $57(19.2 \%)$ influenza positive patients included smoking, lung disease, diabetes, liver disease, heart disease, hypertension, neurologic disease and pregnancy.

\section{Conclusion}

ssInfluenza virus infection was identified as a cause of SARI in hospitalized patients in Vietnam. As a vaccine preventable disease, it remains a burden to the healthcare system as well as a burden to the patient and family. The 0 to $<5$ and 25 to $<35$ age groups were more affected with influenza, accounting for $52.2 \%$ of influenza positive cases. Additional studies may help to better define risk factors related to infection and disease severity, evaluate the use of influenza vaccine in reducing disease burden in Vietnam.

\section{Disclosure of interest}

None declared.

\section{Authors' details}

'Epidemiology, National Institute of Hygiene and Epidemiology, Hanoi, Vietnam. ${ }^{2}$ Virology, National Institute of Hygiene and Epidemiology, Hanoi, Vietnam. ${ }^{3}$ Bill and Melinda Gates Foundation, Seatles, USA. ${ }^{4}$ Program, U.S. Centers for Disease Control and Prevention, Hanoi, Vietnam. ${ }^{5}$ National Institute of Hygiene and Epidemiology, Hanoi, Vietnam.

Published: 16 June 2015

\section{doi:10.1186/2047-2994-4-S1-P13}

Cite this article as: Nguyen et al:: Influenza as a cause of severe acute respiratory infection through sentinel surveillance at national and provincial hospitals in Vietnam. Antimicrobial Resistance and Infection Control 2015 4(Suppl 1):P13. 